Case report

\title{
A Case Report of Duodenal Adenocarcinoma with Diffusely Infiltrative Growth
}

\author{
Hiroshi Nakano, Jun Sasaki, Hiroshi Shimura, \\ Hideaki Nagasaki, Yeng Baek, Yongmu KoH, \\ Gaku Kigawa, Yoshiya Kaneshiro, Toshiyuki Hatakeyama, \\ Sei Takeuchi, Hiroshi Nemoto, Yasuo Yoshizawa, \\ Masahiko Yamaguchi, Kaoru Kumada and Akio NaKayoshi
}

\begin{abstract}
Duodenal adenocarcinoma with diffusely infiltrative growth is a rare tumor. This report presents a patient with a diffusely infiltrative duodenal adenocarcinoma that did not include marked ulceration. The patient's chief complaint was vomiting. The cancer was diagnosed on the basis of an endoscopic biopsy of the tumor. The patient underwent pancreaticoduodenectomy. Pathologic examination showed a duodenal carcinoma with diffusely infiltrative growth. The cancer was diagnosed as an annular-type duodenal adenocarcinoma because of the absence of marked ulceration.
\end{abstract}

Key words : annular-type duodenal adenocarcinoma, diffusely infiltrative growth, pancreaticoduodenectomy

\section{Introduction}

Duodenal adenocarcinoma is a rare malignancy accounting for approximately $0.3 \%$ of all gastrointestinal cancers and approximately $25 \%$ to $40 \%$ of all small bowel cancers ${ }^{1,2)}$. Duodenal carcinomas have been divided on the basis of macroscopic appearance into three types: scirrhous, polypoid, and sessile ${ }^{3)}$. However, the scirrhous type consists mainly of infiltrative cancers with ulceration. Few reports have described diffusely infiltrative duodenal adenocarcinoma in which ulceration is not a significant feature. This report describes a patient with a diffusely infiltrative duodenal cancer without marked ulceration.

\section{Case report}

A 55-year-old man was admitted to another hospital owing to severe nausea and vomiting on May 1, 1996. Duodenal ulcer was diagnosed by means of gastrointestinal endoscopy because of severe stenosis of the duodenum at the postbulbar portion. The patient was referred to our hospital on May 30, 1996, because the symptoms had not improved.

Gastroduodenography performed after admission showed complete obstruction at the second portion of the duodenum (Fig. 1). Gastrointestinal endoscopy showed severe stenosis of the duodenum at the postbulbar portion, and the endoscope could not be passed beyond the obstruction (Fig. 2). Four biopsy specimens were obtained, and undifferentiated 

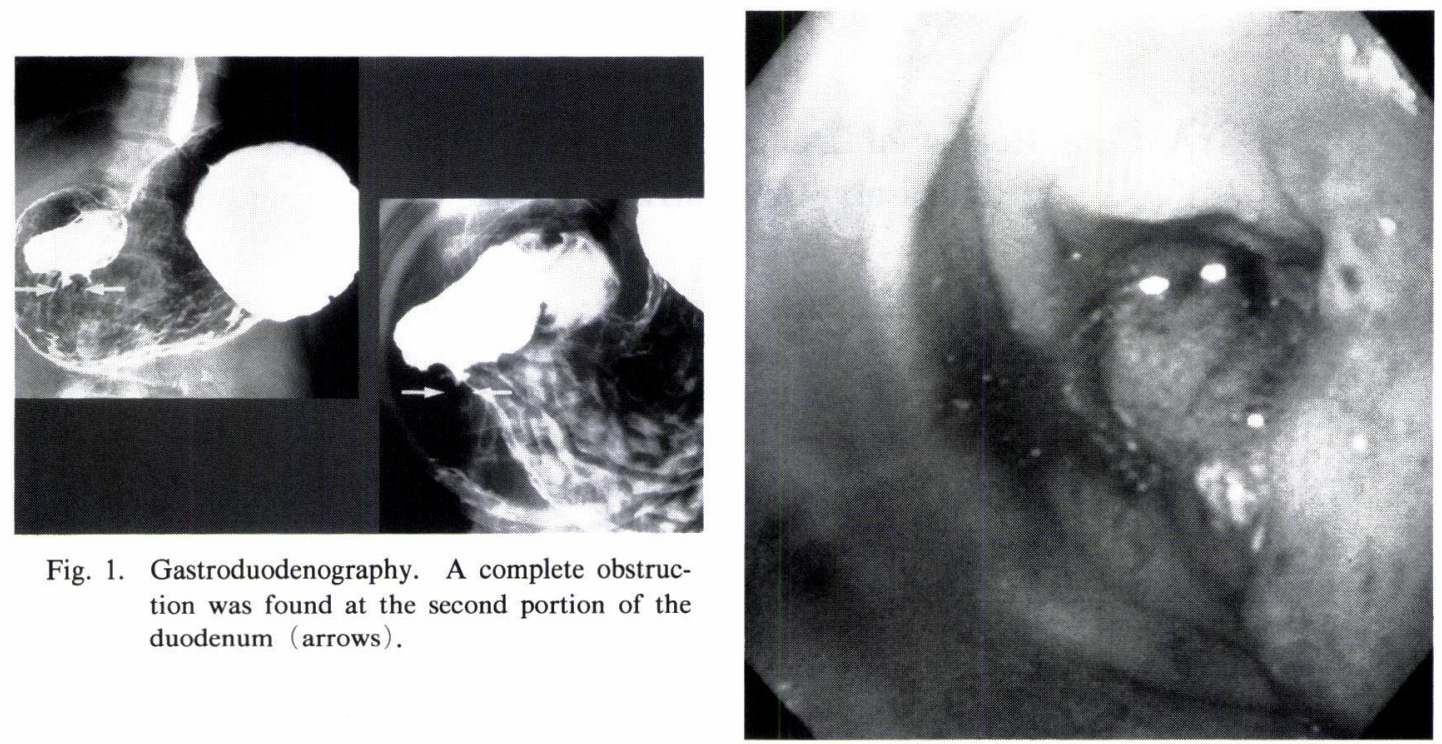

Fig. 1. Gastroduodenography. A complete obstruction was found at the second portion of the duodenum (arrows).

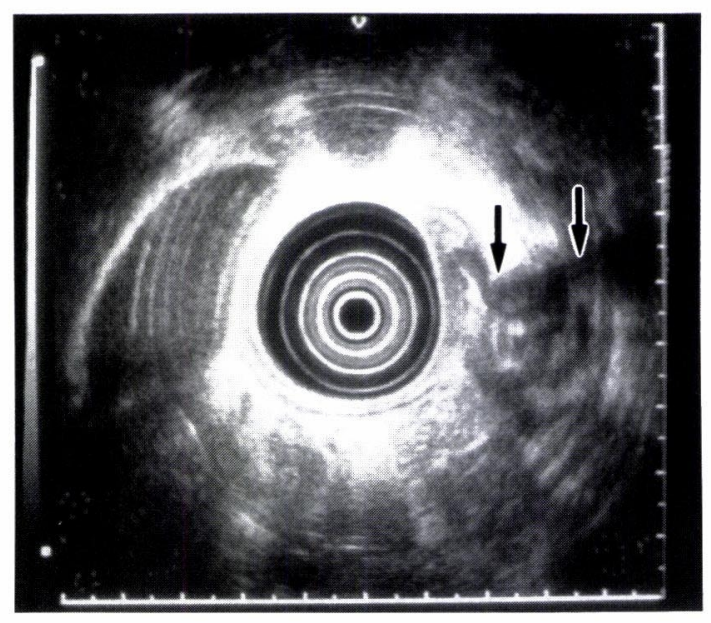

Fig. 3. Echoendosonography at the duodenum. There was an annular hypoechoic mass at the second portion of the duodenum (arrows).
Fig. 2. Gastrointestinal endoscopy at the duodenum. Severe stenosis was confirmed at the postbulbar lesion.

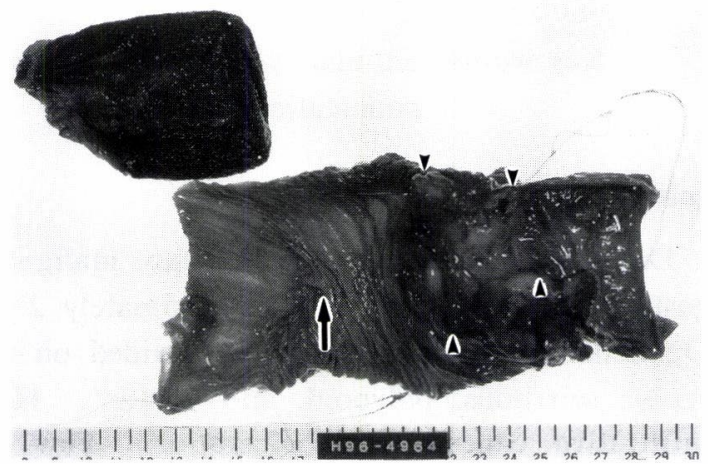

Fig. 4. Macroscopic view of the resected specimen. The cancer lesion did not involve the ampulla of Vater (arrow). The duodenal carcinoma arose between the ampulla and the pylorus and was defined as a supraampullary cancer (arrowheads).

malignant cells were detected in one. Cytokeratin and epidermal membrane antigen specific for adenocarcinoma were detected in malignant cells with specific pathologic staining. Poorly differentiated adenocarcinoma or undifferentiated carcinoma was therefore diagnosed.

Endoscopic ultrasonography showed an annular, diffusely infiltrative tumor of the duodenum (Fig. 3).

Pancreaticoduodenectomy with reconstructive pancreaticogastrostomy and hepaticojejunostomy was performed on June 24, 1996. The annular, diffusely infiltrative carcinoma without marked ulcerations is shown in Fig. 4. The gross appearance of the tumor was classified as type 4 according to Borrmann's classification. The width of the tumor was 


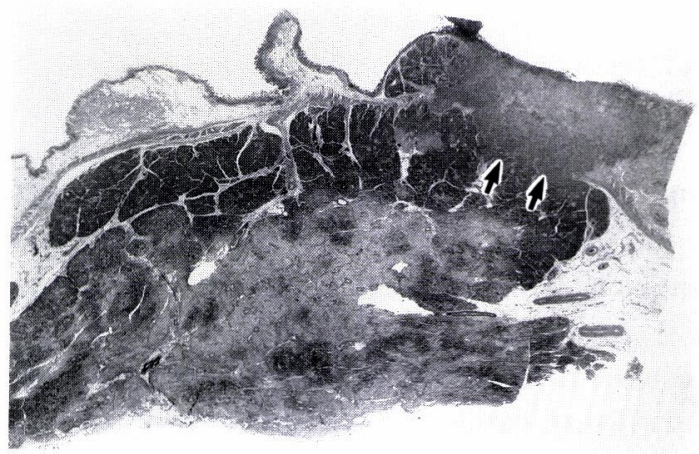

Fig. 5-1. Microscopic view of the resected specimen (low magnification). The cancer diffusely invaded the pancreas (arrows). This diffusely inhiltrative duodenal carcinoma did not show marked ulceration.

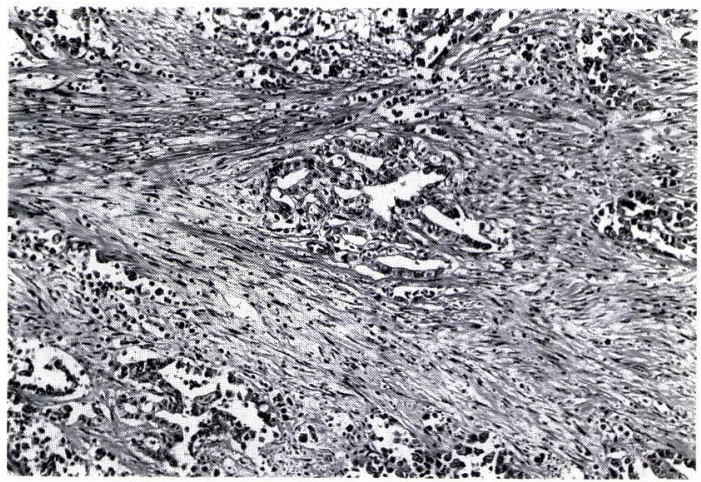

Fig. 5-3. Photomicrograph (original magnification ; $\times 100)$. Tubular formation of the cancer cells was detected in some parts of the tumor. Therefore, the cancer was diagnosed as poorly differentiated adenocarcinoma.

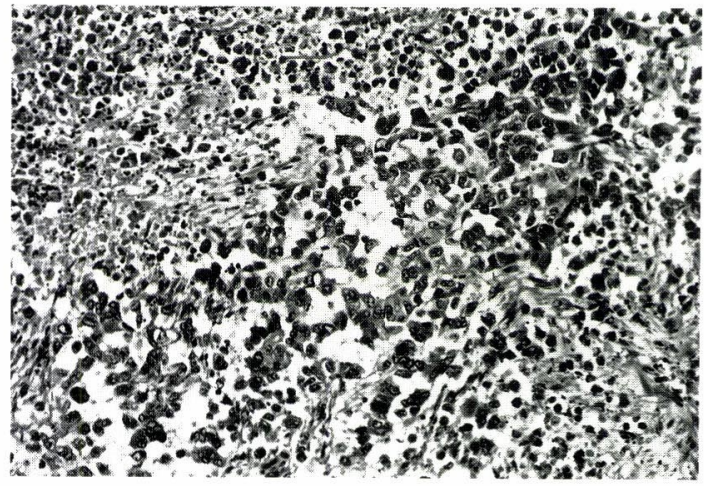

Fig. 5-2. Photomicrogaph (high magnification, original magnification; $\times 200)$. Poorly differentiated adenocarcinoma is shown.

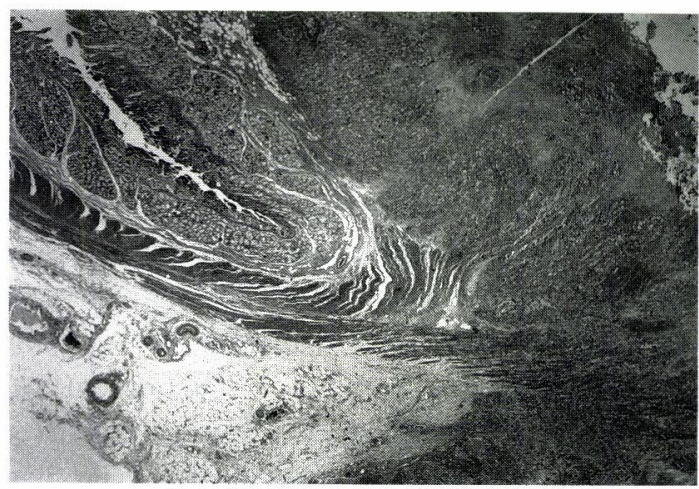

Fig. 5-4. Photomicrograph (original magnification ; $\times 10)$. At the margin of the tumor, pathologic examination showed cancer cell proliferation within the duodenal wall. In addition, the muscularis propriae was destroyed by cancer cell infiltration from the duodenum into the pancreas. Therefore, the cancer was considered to have originated in the duodenum.

$4.5 \times 2.7 \mathrm{~cm}$. The cancer lesion did not involve the ampulla of Vater. Pathologic examination showed pancreatic invasion of undifferentiated cancer cells (Fig. 5-1, 2). Tubular formation of cancer cells was detected in some parts of the tumor (Fig. 5-3). Therefore, the cancer was diagnosed as poorly differentiated adenocarcinoma. At the margin of the tumor, cancer cells had proliferated within the duodenal wall. In addition, the muscularis propriae was destroyed by cancer cell infiltration from the duodenum into the pancreas (Fig. 5-4). Therefore, the cancer was believed to have originated in the duodenum. Metastases were detected to the infrapyloric lymph nodes and the nodes on both the posterior and anterior surfaces of the pancreatic head. The clinicopathologic stage was $\mathbf{T}_{4} \mathbf{N}_{1} \mathbf{M}_{0}$, stage III according to the American Joint Committee on Cancer (AJCC) staging criteria for small intestinal malignancies. These findings indicated that 
pancreaticoduodenectomy was curative.

The patient was alive on July 1, 1997, without recurrence.

\section{Discussion}

Primary malignant neoplasmas of the duodenum are rare. In almost 500,000 autopsies reviewed by Kleinerman and his associates ${ }^{4)}$, the incidence was $0.035 \%$. Iovine and Tsangaris $^{5)}$ found that carcinomas of the duodenum account for $0.35 \%$ of all carcinomas of the gastrointestinal tract.

Duodenal carcinomas have been divided on the basis of macroscopic appearance into three types : scirrhous, polypoid, and sessile ${ }^{3,6}$. The duodenal carcinoma of the present patient was classified as an annular type, which is consistent with type 4 advanced gstric carcinoma according to the Japanese Classification of Gastric Carcinoma ${ }^{7)}$. However, there have been no reports in which the annular type was classified. Moreover, few reports have described diffusely infiltrative duodenal carcinoma in which ulceration is not a marked feature. The scirrhous type consists mostly of ulcerated carcinoma without defined borders, which is compatible with type 3 advanced gastric cancer.

Although a common etiologic factor has been identified in duodenal carcinoma and Gardner's syndrome ${ }^{8)}$, colorectal polyposis was not found in the present patient.

Lillemoe and Imbembo ${ }^{9)}$ reported that duodenal cancers could be divided into four groups on the basis of associated symptoms: obstructing lesions producing early satiety and vomiting; ulcerating lesions causing gastrointestinal bleeding; penetrating lesions invading surrounding tissues and causing gnawing pain; and lesions encroaching upon the ampulla of Vater and causing jaundice. The present patient had an obstructing lesion.

Neoplasmas of the duodenum are usually classified according to their location relative to the ampulla of Vater. Duodenal carcinomas arising between the ampulla and the pylorus are referred to as supra-ampullary. Those arising from the mucosa surrounding the ampulla are defined as periampullary, while those between the ampulla and the ligament of Treitz are infra-ampullary. Spira et al. ${ }^{1)}$ reported that $56.3 \%$ of duodenal carcinomas were infra-ampullary in origin, $32.4 \%$ periampullary, and only $7.0 \%$ supra-ampullary. Therefore, the duodenal cancer of the present patient which was defined as a supraampullary lesion is considered to be rare.

Fortunately, the duodenal carcinoma of the present patient was diagnosed on the basis of biopsy specimens obtained at the first gastrointestinal endoscopy after admission. If the cancer had not been confirmed, we would have performed endoscopic surface-brushings as recommended by Lillemoe and Imbembo ${ }^{9)}$.

Few studies have examined the effect of staging on outcome in patients with duodenal carcinoma. Ouriel and Adams advocated the use of the modified Duke's system of Astler and Coller in the staging of small intestinal adenocarcinomas ${ }^{10)}$. Recently, Rose et al. ${ }^{11)}$ reporeted that the AJCC staging criteria for small intesinal carcinomas is valuable and well correlated with survival rate in cases of duodenal carcinoma. The clinicopathologic stage of the present patient according to the AJCC criteria was stage III, which Rose et al. reports to have a 4-year survival rate of approximately $40 \%{ }^{11}$.

Pickleman et al. recently reported the favorable prognosis in cases of duodenal adenocarcinoma with lymph node metastasis ${ }^{12)}$. Of seven duodenal carcinoma patients with lymph node metastasis who were treated with pancreaticoduodenectomy, 4 survived for at 
least 5 years. They concluded that pancreaticoduodenectomy may be curative for duodenal carcinoma even if lymph node metastasis is present.

In conclusion, the diffusely invasive duodenal adenocarcinoma without marked ulceration is considered to be very rare. We reported a case in which resection was curative.

\section{References}

1) Spira IA, Ghazi A and Wolff WI : Primary adenocarcinoma of the duodenum. Cancer 39: 1721-1726 (1977)

2) Alwmark A, Anderson A and Lasson A : Primary carcinoma of the duodenum. Ann Surg 191: 13-18 (1980)

3) Cortese AF and Cornell GN : Carcinoma of the duodenum. Cancer 29: 1010-1015 (1972)

4) Kleinerman J, Yardumian K and Tamaki HT : Primary carcinoma of the duodenum. Ann Intern Med 32 : 451 $-455(1950)$

5) Iovine VM and Tsangaris N : Primary carcinoma of the duodenum. Am J Surg 27: 744-747 (1961)

6) Pascal RR, Perzin KH and Fenoglio-Preiser CM: Neoplastic diseases of the small and large intestine. In : Principles and Practice of Surgical Pathology, Silverberg SG (ED), 2nd edition, Churchill Livingstone, New York, pp 1185-1240 (1990)

7) Japanese Research Society for Gastric Cancer: Macroscopic types. In: Japanese Classification of Gastric Carcinoma. 1st English edition, Kanehara, Tokyo, pp 4-5 (1995)

8) Schnur PL, David E, Brown PW Jr, Beahrs OH and Harrison EG Jr: Adenocarcinoma of the duodenum and the Gardner syndrome. JAMA $223: 1229-1232$ (1973)

9) Lillemoe K and Imbembo AL: Malignant neoplasms of the duodenum. Surg Gynecol Obstet 150: 822-826 (1980)

10) Ouriel K and Adams JT: Adenocarcinoma of the small intestine. Am J Surg 147: 66-71 (1984)

11) Rose DM, Hochwald SN, Klimstra DS and Brennan MF: Primary duodenal adenocarcinoma : a ten-year experience with 79 patients. J Am Coll Surg 183: 89-96 (1996).

12) Pickleman J, Koelsch $M$ and Chejfec G : Node-positive duodenal carcinoma is curable. Arch Surg 132 : 241244 (1997).

[Received July 29, 1997 : Accepted December 25, 1997] 